\title{
Deformation processes near a crack initiation site under dwell-fatigue loading of Ti-6Al-4V
}

\author{
C. Lavogiez ${ }^{1}, \mathrm{~S}$. Hémery ${ }^{1, *}$, P. Villechaise ${ }^{1}$ \\ ${ }^{1}$ Institut Pprime, CNRS-ENSMA, Université de Poitiers, UPR CNRS 3346, Physics and Mechanics of Materials Departement, \\ ENSMA - Téléport 2, 1 avenue Clément Ader, BP 40109, 86961 Futuroscope Chasseneuil Cedex, France \\ *samuel.hemery@ensma.fr
}

\begin{abstract}
:
The present article reports an investigation of the mechanism of surface crack initiation of a dwell fatigue tested Ti-6Al-4V alloy with a bi-modal microstructure. Interactions between slip bands and grain boundaries were characterized in order to obtain insights into the crack initiation process and discuss the similarities with models described in the literature. Twinning and local lattice rotation occurred as a result of the slip band blocking at the interface and suggests high local stress concentrations. Nevertheless, crack initiation happened to be intergranular and not transgranular. The crack opened up the basal plane that was located at the interface between two nodules poorly oriented for slip and having a common c axis of the hexagonal unit cell.
\end{abstract}

\section{Introduction}

Owing to superior mechanical properties for a relative lightweight and good corrosion resistance, titanium alloys are widely employed in the aeronautic industry [1]. For aero-engine applications, titanium alloy are exposed to fatigue loading including hold time at maximum stress (i.e. also denoted as dwell-fatigue). Due to the known capacity of titanium alloys to creep at room temperature, early works of Evans and Goteslow [2] revealed a fatigue life debit of IMI 685 if a load hold at maximum stress is introduced. As it is commonly agreed that the majority of the fatigue life of titanium alloys is consumed by the crack initiation stage, many efforts were put into the understanding of dwell fatigue crack initiation. Nevertheless, as opposed to fatigue loading, subsurface crack initiation occurs under dwell fatigue loading [3]. Quasi cleavage facets with a normal parallel to the loading direction were found near the crack initiation site on the fracture surface. The quasi-cleavage plane is basal or near basal [4]. Based on these observations, Evans and Bache suggested a modified version of Stroh's model [5]. According to the authors, crack initiation is a consequence of basal or prismatic slip operating in an adjacent grain (denoted as "soft" grain in the following).The dislocations piledup at the boundary with the hard grain will generate the required stress state to induce facet formation. This model has been deeply investigated using crystal plasticity simulations [6-8] and completed to include time dependency. A key contribution comes from soft regions off-loading stress on to hard regions (small misorientation between $\mathrm{c}$ axis and loading direction). Indeed, the difficult deformation of the $\alpha$ Ti hexagonal closed packed lattice along the $<\mathbf{c}>$ axis, which is implied by a high critical resolved shear stress (CRSS) for pyramidal slip [9] and twinning inhibition due to a high aluminum content [1], leads to important stress and strain heterogeneities. As a result, load shedding occurs in the regions with low plastic strain accumulation (i.e the hard grains) [10]. The mean stress magnitude in the hard grain is then increased. Adding a local stress concentration, the blocked dislocation pile up at the hard grain interface as described by Evans and Bache, may then trigger crack initiation [6,7]. However, recent experimental works revealed that the crystallographic orientation of nodules where crack initiation proceeds overlaps with the orientation domain associated with the operation of basal slip [11]. This seems in contradiction with the "hard" grain where crack initiation is supposed to proceed according to the Evans and Bache model. In this context, the main motivation of this work is to investigate the deformation processes occurring near crack initiation sites under dwell fatigue loading. In this aim, a specimen of Ti-6Al-4V with a bi-modal microstructure has been dwell fatigue tested until failure. An initiated crack, and the deformation processes operating in the neighboring region, was thoroughly characterized in order to understand the crack initiation process.

\section{Materials and experiment}

The material of interest is the Ti-6Al-4V alloy with a bi-modal microstructure (Figurela). This duplex microstructure is composed of $\alpha_{\mathrm{p}}$ nodules and colonies of $\alpha_{\mathrm{S}}$ lamellas precipitated in a $\beta$ matrix. According to EBSD analyses, the equivalent diameter of the nodules 
is about $13 \mu \mathrm{m}$ and the associated surface fraction is $36 \%$. The crystallographic texture of the sample is represented on the inverse pole figure coded following the loading direction on Figure 1b. The maximum value of the orientation distribution function is 1.65 . This indicates a weak texture of the sample.

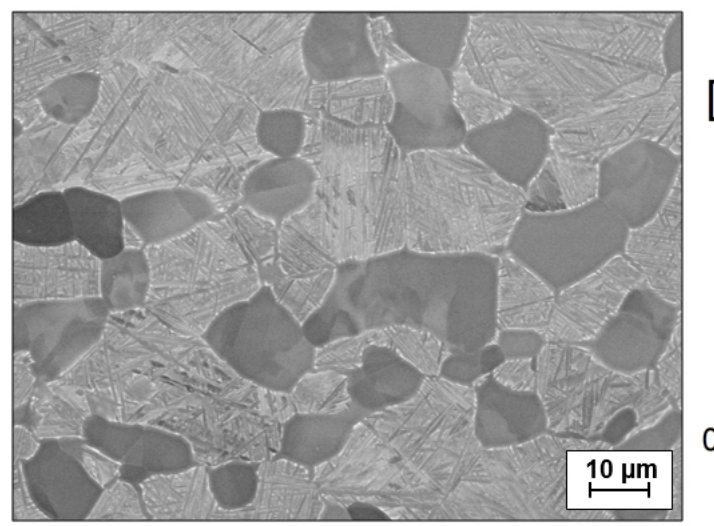

\section{[010]}

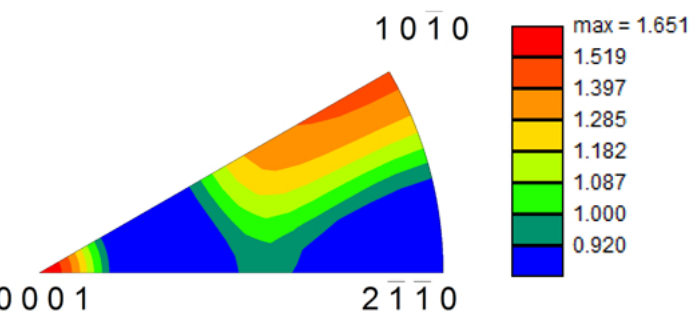

Figure 1 : a. SEM micrograph of the bimodal microstructure b. Inverse pole figure of the orientation distribution function with respect to the loading direction.

The gage length of the specimen used for mechanical testing is $10 \mathrm{~mm}$ long, $2 \mathrm{~mm}$ wide and $1 \mathrm{~mm}$ thick. The sample geometry, with a rectangular cross section, has been chosen to facilitate the SEM characterization of the deformation and crack initiation processes. Prior to the mechanical testing careful surface preparation of the sample has been done to allow SEM characterization thereafter. The samples were grinded and polished with colloidal silica suspension (OP-S) with $10 \% \mathrm{H}_{2} \mathrm{O}_{2}$ as the final step. The mechanical testing were performed on an INSTRON 1362 testing machine under load control using a trapezoidal loading waveform. The maximum stress was fixed to $0.85 \mathrm{x}$ yield stress. The yield stress was previously estimated using a dedicated tensile test with an applied strain rate equivalent to the loading rate of the cyclic tests. The time at maximum stress has been set to $120 \mathrm{~s}$ to simulate the dwell fatigue loading condition. In order to be as representative as possible of the in service loading condition, the stress ratio is positive and equal to 0.1 . The loading and unloading steps were performed in $1 \mathrm{~s}$ and the time at minimum stress is $1 \mathrm{~s}$ as well. Finally, the specimens were tested until failure of the samples.

Afterwards, a JEOL 6100 scanning electron microscope was employed to characterize the deformation processes using a slip traces analysis. This microscope is equipped with an OIM setup provided by EDAX to acquire the local crystallographic orientations (EBSD analyses).

\section{Results}

\subsection{Microstructural crack initiation site description}

The micrograph of Figure 2 shows a secondary crack detected on the sample surface. The crack initiated in grain 2 and propagated in grain 1 and 3. Several straight slip traces are detected on surface of G1. According to the slip trace analysis, they testify of basal slip activity with an apparent Schmid factor of 0.402. The slip traces impinge the interface with G2-1 but slip transfer does not seem to occur as no active deformation system is detected in G2. In G3, slip traces with two different orientations are observed. The slip traces reveals the operation of basal slip with a Schmid factor of 0.430 and 1st order pyramidal slip with a Schmid factor of 0.429 assuming the slip proceeds along an direction. Indeed, a lower CRSS is reported for type pyramidal slip than type pyramidal slip. The slip traces of both slip systems impinge again the interface with G2-1. Again, slip transfer does not seem to occur. G2 has the lowest angle between the $\mathrm{c}$ axis of the hexagonal unit cell and the loading direction $\left(\approx 16^{\circ}\right)$. The maximum apparent Schmid factor for basal slip is 0.26 which is lower than for G1 and G3, which are 0.402 and 0.430 respectively. 


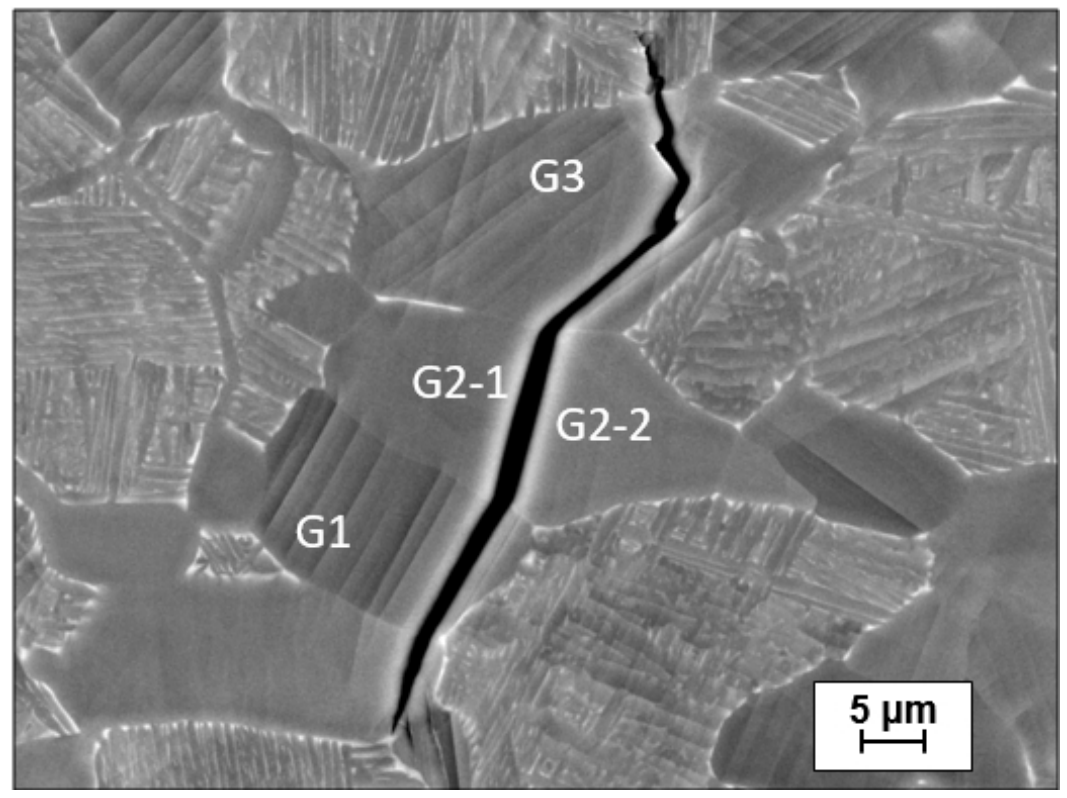

Figure 2: SEM micrograph of crack intiation on surface of the dwell fatigue sample.

\subsection{Slip band / grain boundary interactions}

The crystallographic orientation in the crack initiation region is shown on an inverse pole figure map with the index quality superimposed (Figure 3a). The grain reference orientation deviation map, which is calculated relative to the average orientation of each grain, is shown on Figure 3b. The inverse pole figure map reveals the presence of a twin within grain G2 close to the interface with the upper grain G3. According to the crystallographic orientation relationship, the twinning system is (-1012) [10-11]. The twin has a lenticular shape with a root localized at the grain boundary. This let suppose that twinning was stimulated by a slip band in G3 impinging the grain boundary as previously reported in the literature [12,13]. At the interface between G2 and G1 such twin activation is not detected. Rather, the grain reference orientation deviation map reveals the presence of local lattice rotations induced by slip bands blocking at the G1/G2 interface. These lattice rotations are mostly of low magnitude at the exception of the one circled one on Figure $3 \mathrm{~b}$. The magnitude of the lattice rotation is of $5.4^{\circ}$ and progressively vanishes as the distance from the head of the incoming slip band increases. The high magnitude of lattice rotation suggests here high local stress concentration in G2 due to a dislocation pile up at the interface. At the upper interface G2/G3, such high local lattice rotation gradient are not observed. Twinning might have helped in relaxing the local stress concentration due to accommodation of the strain incompatibilities. 


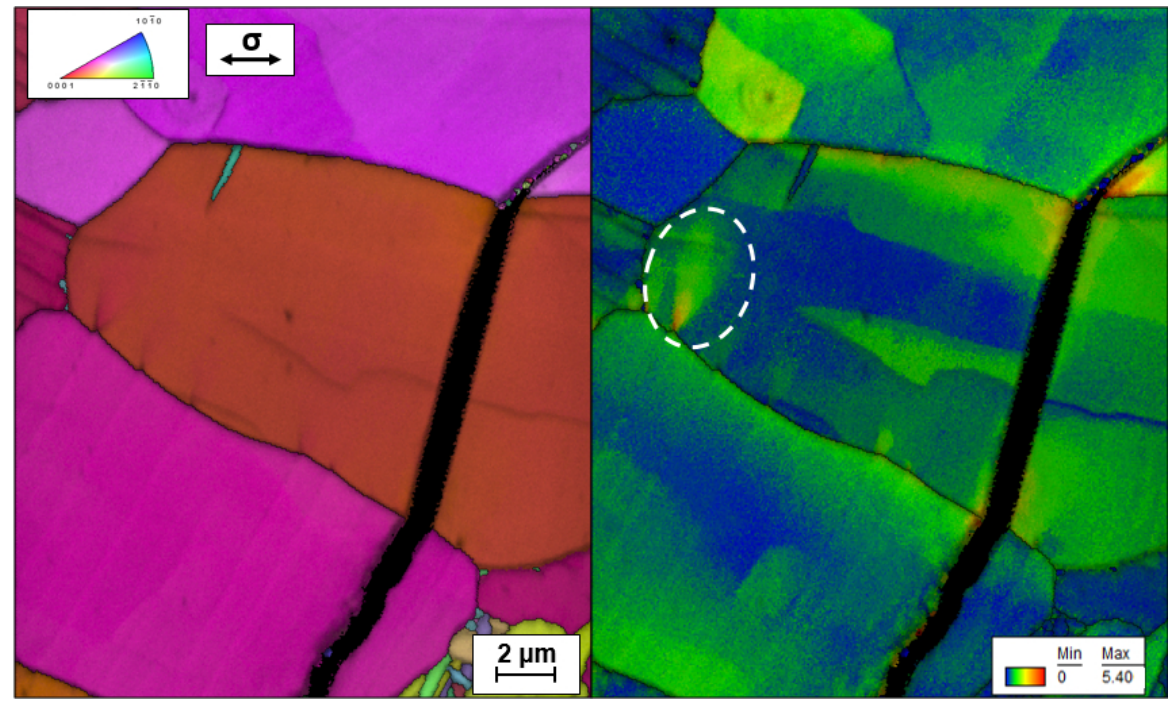

Figure 3 : a. Crystallographic orientation map (a.) and grain reference orientation deviation map (b.) of the micrograph shown in figure $3 a$.

In the attempt to understand the condition of twinning, the Schmid factor and m' parameter were characterized for both interface. The $\mathrm{m}$ ' parameter characterizes the crystallographic alignment between the incoming and outgoing deformation system across a grain boundary [14]. Its influence has been reported in the literature for slip induced twinning in CP-Ti [13]. The interface between G2 and G3 where twinning occurred will be firstly considered. The Schmid factor and m' parameter values are reported in table 1. The two slip system of G3 are considered as the incoming slip system in order to calculate two sets of m' values. The outgoing deformation system is kept constant as the twinning variant activated that was identified. In table1, the Schmid factor of the incoming slip systems are recalled. Both are roughly equal to 0.43 , giving a higher interest on the m' parameter value. The Schmid factor of the observed twin system is 0.45 . The m' parameter considering the basal slip system of G3 as the incoming slip system and the twinning system of G2 as the outgoing deformation system is 0.92 . This high value evidence a good crystallographic alignment between the two deformation systems. The $\mathrm{m}$ ' parameter considering now the $1^{\text {st }}$ order pyramidal slip system of G3 as the incoming slip system and the twinning system in G2 as the outgoing deformation system is 0.46 , hinting a poor alignment. As Wang and coworker [13] reported slip induced twinning at high m' parameter values, it may suggest that twinning was stimulated by the basal slip system of G3.

Table 1 : Schmid factors and $m$ ' values considering the deformation system at the G3/G2-1 interface

\begin{tabular}{|l|l|r|l|r|r|}
\hline & \multicolumn{2}{|c|}{ Incoming } & \multicolumn{2}{c|}{ Outgoing } & \\
\hline Interface & Slip sys tem & Schmid factor & Twinning sys tem & Schmid factor & m' factor \\
\hline G3/G2-1 & B3 $(0001)[-2110]$ & 0,430 & T3 (-1012) $[10-11]$ & 0,453 & 0,923 \\
\hline G3/G2-1 & Pi<a $>6(1-10-1)[11-20]$ & 0,429 & T3 (-1012) $[10-11]$ & 0,453 & 0,462 \\
\hline
\end{tabular}

In table 2, the interface G1/G2 is of interest. As only one slip system is observed in G1, there is no indetermination on the incoming deformation system. The incoming deformation system is thus the basal slip system with a Schmid factor of 0.40 . However, as twins are not observed, all the 6 twinning variants are considered. The Schmid factors of the twinning system are compromised in the range $\left[0.40 ; 0.46\left[\right.\right.$. The variant with the best alignment $\left(\mathrm{m}^{\prime}=0.88\right)$ with the incoming basal slip system is T3. Although good crystallographic alignment of $\mathrm{T} 3$ is noticed toward both the incoming deformation system and loading direction, it was not observed. The variant T3 is precisely the one activated at the upper interface between G2/G3 with a similar m' values (0.92). As a result, m' 
and the Schmid factor are not sufficient to predict whether twinning will occur or not here since the value of m' and of the Schmid factors are close to each other for both two interfaces and does not allow to differentiate the two interactions.

Table 2 : Schmid factors and $\mathbf{m}$ ' parameters considering the deformation system at the interface G1/G2-1

\begin{tabular}{|c|c|c|c|c|c|}
\hline & \multicolumn{2}{|c|}{ Incoming } & \multicolumn{2}{|c|}{ Outgoing } & \multirow[b]{2}{*}{ m' factor } \\
\hline Interface & Slip system & Schmid factor & Deformation system & Schmid factor & \\
\hline \multirow{9}{*}{ G1/G2-1 } & \multirow{9}{*}{ B1 (0001) [11-20] } & \multirow{9}{*}{0,402} & B1 (0001) [11-20] & 0,175 & $0,0,507$ \\
\hline & & & \begin{tabular}{|l|}
$\mathrm{B} 2(0001)$ [1-210] \\
\end{tabular} & 0,265 & 0,265 \\
\hline & & & B3 (0001) [-2110] & 0,090 & 0,772 \\
\hline & & & T1 (-1102) [1-101] & 0,454 & 0,724 \\
\hline & & & T2 (1-102) [-1101] & 0,418 & 0,077 \\
\hline & & & T3 (-1012) [10-11] & 0,453 & 0,880 \\
\hline & & & T4 (10-12) [-1011] & 0,462 & 0,108 \\
\hline & & & T5 (0-112) [01-11] & 0,402 & 0,305 \\
\hline & & & T6 (01-12) [0-111] & 0,446 & 0,118 \\
\hline
\end{tabular}

\subsection{Crack initiation}

The crack observed on the micrograph of Figure 2 propagated in both G1 and G3. The crack trace is consistent with the basal plane for $\mathrm{G} 1$ and a $1^{\text {st }}$ order pyramidal plane for G3 that also corresponds to the slip system activated in both grains. The crack in G2 corresponds to the basal plane trace. The orientation of the basal plane considering the orientation G2-1 and G2-2 is presented on Figure 4a. Nevertheless, the prismatic planes do not have the same orientation between G2-1 and G2-2 as revealed by the direct pole figure in Figure 4a. This indicates a misorientation between G2-1 and G2-2 around the c axis. The misorientation profile perpendicular to the crack at it initiation site is shown on Figure $4 \mathrm{~b}$. It reveals a misorientation value of $20^{\circ}$ that is too high to result from crack opening. It thus indicates the presence of a grain boundary at the crack initiation site. Furthermore, this interface corresponds to the basal plane trace. Thus, the crack initiation seems here to be intergranular.
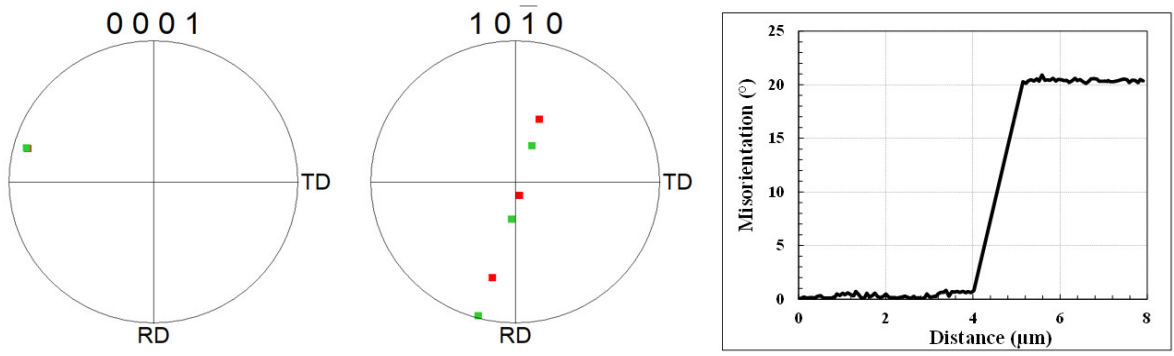

Figure 4 : a. Crystallographic orientation of the basal (0001) and prismatic (10-10) plane of G2-1 and G2-2 represented on direct pole figure and b. misorientation profile across the crack of Figure 3a from G2-1 to G2-2.

\section{Discussion}

The crack investigated displays several characteristic of both fatigue crack initiation and dwell fatigue crack initiation taken from the literature. Firstly, the surface initiation is an attribute of fatigue crack initiation. In the work of Bridier and coworker, fatigue crack initiations are associated with either prismatic or basal slip activity [15]. Crack initiation in the basal slip orientation domain occurs in nodules with an angle between the $\mathrm{c}$ axis and loading direction as low as $15^{\circ}$. The orientation of the nodules at crack initiation in the present work with an angle of $16^{\circ}$ matches this fatigue crack orientation domain. Nevertheless, basal slip was not detected on the micrograph within the crack initiating nodules. Crack initiation seemingly occurred along the basal plane of G2-1 and G2-2 in 
agreement with the quasi-cleavage crack initiation reported in the literature. In addition, the microstructural arrangement of the investigated crack has the characteristic of the hard and soft grain pair of the modified Stroh model [5,6] for dwell fatigue facet formation. In addition to the crack initiating nodules satisfying the hard grain definition, they are surrounded by two nodules with an intense plastic slip activity. As a consequence load shedding as described in simulation works [6,7,10] should be occurring from G1 and G3 to G2-1 and G2-2, increasing the magnitude of the stress. The local stress concentration resulting from the dislocation pile up as evidenced by local lattice rotation, might have then triggered crack initiation. The intergranular character of the crack is a new parameter to the crack initiation mechanism described in the literature for both fatigue and dwell fatigue to the author knowledge, although the main features of the Stroh model still hold. The crack path seems to follow the pyramidal plane in G3 and the basal plane in G1 that are consistent with the active slip systems in those grains.

If twinning does not occur, the dislocation pile up due to an incoming slip band to the interface of G2-1 lead to a lattice rotation gradient that shows very similar characteristics to the so called micro-volume observed in Ni-based superalloys. The micro volumes were shown to be at the origin of fatigue crack initiation at the grain boundary due to the high stress induced [16]. Such micro volume was not observed near in the second interface where incoming slip bands impinge the interface of G2-1. Rather the blocked slip band seems to have induced twinning as also reported in CP-Ti. In CP-Ti Wang and coworker [13] have highlighted a satisfying twinning prediction using the $\mathrm{m}$ ' parameter. Nevertheless, the $\mathrm{m}$ ' values are similar for basal slip grain boundary interaction for G2-1 between the lower and the upper interface (Figure 3a). Slip induced twinning in titanium might thus be the result of complex interactions that the crystallographic alignment cannot solely account for. In addition, due to the stress concentration induced by the dislocation pile up, the stress state might deviate from uniaxial. As the Schmid factor is computed based on homogenous uniaxial stress state assumption, it appears too limited for the analysis of slip induced twinning. Nevertheless, the variant activated did correspond to the one with the maximum m' value confirming partly the result of Wang and coworker in case of Ti-6Al-4V. Thus, nodules where crack initiation proceeds can possibly be concerned by twinning. However, no twin was noticed near the crack and suggest that twinning is not directly involved in crack initiation processes.

\section{Conclusion}

The presented work has investigated the mechanism of deformation at a crack initiation site on surface of a sample loaded in dwell fatigue. A secondary crack was noticed on the surface of the specimen and was thoroughly analyzed. The crack has initiated at the interface following a basal plane between two nodules sharing a common c axis of the hexagonal unit cell. Basal slip was not detected in the crack initiating nodule, in agreement with the Evans and Bache model. The interaction of adjacent basal slip with the hard grain boundary lead to either twinning or local lattice misorientations indicating a high stress concentration. Twinning does not seem directly involved in the crack initiation process and rather seem to enable accommodation of local stress concentrations.

\section{References}

[1] G. Lütjering, J.C. Williams, Titanium, Springer Science \& Business Media, 2007.

[2] W.J. Evans, C.R. Gostelow, Metall. Trans. A (n.d.) 10.

[3] M. Bache, Int. J. Fatigue 25 (2003) 1079-1087.

[4] M.R. Bache, W.J. Evans, H.M. Davies, (n.d.) 8.

[5] W. Evans, M. Bache, Int. J. Fatigue 16 (1994) 443-452.

[6] F.P.E. Dunne, D. Rugg, A. Walker, Int. J. Plast. 23 (2007) 1061-1083.

[7] Z. Zhang, M.A. Cuddihy, F.P.E. Dunne, Proc. R. Soc. Math. Phys. Eng. Sci. 471 (2015) 20150214.

[8] M. Anahid, M.K. Samal, S. Ghosh, J. Mech. Phys. Solids 59 (2011) 2157-2176.

[9] I.P. Jones, W.B. Hutchinson, Acta Metall. 29 (1981) 951-968. 
[10] V. Hasija, S. Ghosh, M.J. Mills, D.S. Joseph, Acta Mater. 51 (2003) 4533-4549.

[11] A.L. Pilchak, J.C. Williams, Observations of Facet Formation in Near-a Titanium and Comments on the Role of Hydrogen:, Defense Technical Information Center, Fort Belvoir, VA, 2010.

[12] C. Lavogiez, S. Hémery, P. Villechaise, Scr. Mater. 157 (2018) 30-33.

[13] L. Wang, Y. Yang, P. Eisenlohr, T.R. Bieler, M.A. Crimp, D.E. Mason, Metall. Mater. Trans. A 41 (2010) 421430.

[14] J. Luster, M.A. Morris, Metall. Mater. Trans. A 26 (1995) 1745-1756.

[15] F. Bridier, P. Villechaise, J. Mendez, Acta Mater. 56 (2008) 3951-3962.

[16] B. Larrouy, P. Villechaise, J. Cormier, O. Berteaux, Acta Mater. 99 (2015) 325-336. 